\title{
Watermarking based on Difference Expansion and Genetic Algorithms
}

\author{
Laura M. Vargas
}

\begin{abstract}
Security problems that recently appeared in transmission and storing of digital content can be solved by using watermarking. Reversible watermarking is the election in medical and defense imagery because these security systems are able to restore the original image after extracting the hidden information. In this document an intelligent reversible watermarking scheme is proposed. It is based on the difference expansion method developed by Tian and it is helped by genetic algorithms to improve the imperceptibility of the mark for a fixed payload. Furthermore, it is shown that the image can be divided in blocks watermarking each one, so if an adulteration takes place, it can be determined the block where this happened.
\end{abstract}

Keywords-reversible watermarking, Haar transform, difference expansion, genetic algorithms, information security.

\section{Introduction}

During the last two decades various schemes of watermarking have been developed to protect digital data from illegal use. In recent years attention was put on lossless data hiding for protecting and authenticating medical and military data. Digital watermarking introduce distortion in the host: more capacity the scheme has, less imperceptibility of the watermark. The solution for legal applications is that the final user could work on the original file. Nevertheless the marked image must have a good quality, enough for common users. Fig. 1 shows the scheme of reversible watermarking.

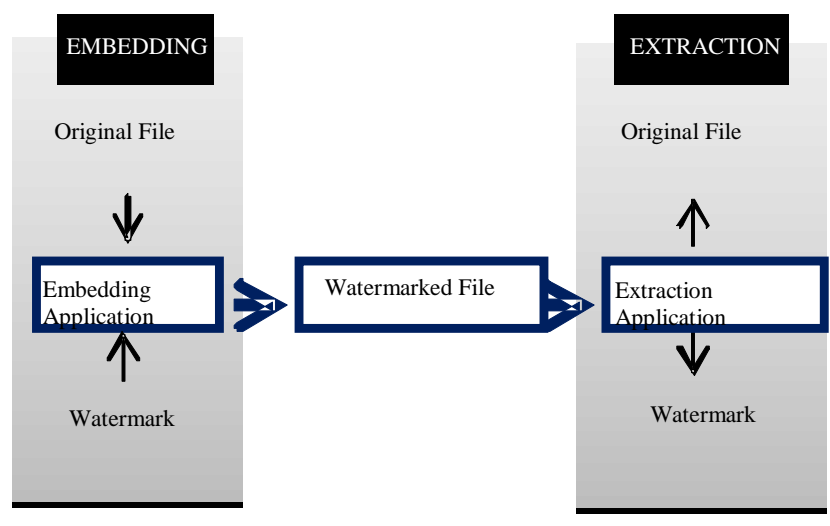

Figure 1. Scheme of reversible watermarking

Laura M. Vargas

Universidad Nacional de Córdoba

Argentina
In conventional watermarking only the secret message is recovered, the file remains watermarked. A trade-off between capacity and quality of the algorithm must be achieved for both types of schemes, so they can be useful. Many reversible watermarking have been proposed. Ni's algorithm is based on the displacement of the histogram [1]. This technique has high quality but the capacity depends on the histogram peak value. Xuan's algorithm [2] embeds the secret data in the reversibly compressed middle plane of the IWT (Integer Wavelet Transform) domain with an acceptable capacity. Tian's algorithm [3] calculates differences between pairs of adjacent pixels and chooses some of them to embed the watermark after displacing the byte one bit to the left. This displacement is called Difference Expansion (DE). This algorithm has good quality and capacity.

In this paper a reversible algorithm based on the Tian's method of DE is presented. The proposal includes the use of genetic algorithms to achieve capacity without losing the imperceptibility of the watermark in the host image.

\section{Difference Expansion: Original Tian's Algorithm}

Tian presented his algorithm based on difference expansion in 2003. The watermark is embedded in the Wavelet Integer Transform domain. The algorithm takes the pixels in pairs without overlapping, and the mean and difference of the pixels are calculated. Equations (1) and (2) show the transformation, that is clearly reversible.

$$
\begin{gathered}
l=\left\lfloor\frac{a+b}{2}\right\rfloor \\
h=b-a
\end{gathered}
$$

where $a$ and $b$ are the intensity pixel values.

Tian proposed to expand the difference, displacing to the left the bits of $h$ one bit (this is equivalent to duplicate $h$ ) and then adding $b$. To prevent overflow and underflow problems, $h$ must satisfy (3) after the embedding, where $\mathrm{n}$ is the number of bits per pixel (i.e. 8 if the image has 8 bits per pixel).

$$
|h| \leq \min \left(2\left(<\left(2^{n}-1\right)-l\right), 2 l+1\right)
$$

The theoretical maximum capacity is equal to 0,5 bits per pixel. In 2007 Weng proposed taking quads and embedding the watermark in three differences called $v$ [4]. This is shown in (4) and (5). The maximum theoretical capacity increases up to $0,75 \mathrm{bpp}$. Watermark bits $b_{k}, k$ belonging to interval $(1, \mathrm{~L})$ 
where $\mathrm{L}$ is the length of the mark, are added to the expanded differences (6), obtaining the watermarked differences $\tilde{v}$.

$$
\begin{aligned}
& {\left[\begin{array}{ll}
u_{0} & u_{1} \\
u_{2} & u_{3}
\end{array}\right] \stackrel{T}{\rightarrow}\left[\begin{array}{ll}
v_{0} & v_{1} \\
v_{2} & v_{3}
\end{array}\right]} \\
& v_{0}=\left\lfloor\frac{u_{0}+u_{1}+u_{2}+u_{3}}{4}\right\rfloor \\
& v_{1}=u_{0}-u_{1} \\
& v_{2}=u_{0}-u_{2} \\
& v_{3}=u_{0}-u_{3} \\
& \tilde{v}_{i}=2 v_{i}+b_{k} \quad i=1,2,3
\end{aligned}
$$

In a similar way Alattar [5] constructs a vector of $n$ pixels, calculates the mean and $(n-1)$ differences which are embedded if they don't cause overflow or underflow. In this method the maximum theoretical capacity is equal to $(n-1) / n$ bpp. but the imperceptibility of the mark is affected as $n$ increases.

Weng's algorithm proposes a trade-off between capacity and quality of the system. To avoid the problem of overflow or underflow or at least to reduce it, there's a technique called compansion. The differences over a threshold called $T$ are compressed (see Fig. 1). This compression needs to be reverted when the original image is recovered. All these steps add an extra payload, i.e. information that must be embedded with the payload o watermark to recover the original image. The equations (7) and (8) show how the compression and expansion of the differences are implemented.

$$
\begin{gathered}
C(v)=v_{c}= \begin{cases}v & |v|<T \\
\left.\operatorname{sign}(v) \times\left(\mid \frac{|v|-T}{2}\right\rfloor+T\right) & |v| \geq T\end{cases} \\
E(C(v))=E\left(v_{c}\right)= \begin{cases}v_{c} & \left|v_{c}\right|<T \\
\operatorname{sign}\left(v_{C}\right) \times\left(2 \times\left|v_{C}\right|-T\right) & \left|v_{c}\right| \geq T\end{cases}
\end{gathered}
$$

Because of the digital character of the intensity, the floor function meaning "the greatest integer less or equal to" must be used in the division. Then, an error indicated in. (9) may be produced and must be stored to assure the reversibility of the process.

$$
\text { Error }=e=v-|E(C(v))|
$$

Not only the threshold and the compansion errors must be stored but also the map indicating the positions of the embedded differences. So, the watermark is composed at least by the imperative following fields:

M: map of the differences that contain the bits of the mark

E: compansion errors

W: watermark
The map and the compansion errors are subjected to arithmetical compression to save space.

The compression threshold controls the capacity and the quality of the scheme. In watermarking the quality is valuated using MSE (Mean Square Error) (10) or PSNR (Peak Signal Noise Ratio) (11).

$$
\begin{gathered}
M S E=\frac{1}{M x N} \sum_{i=1}^{M} \sum_{j=1}^{N}\left(I_{W}(i, j)-I(i, j)\right)^{2} \\
P S N R(d B)=10 \log _{10} \frac{\left[I_{\text {peak }}\right]^{2}}{M S E}
\end{gathered}
$$

For an image with $\mathrm{M}$ rows, $\mathrm{N}$ columns, $I(i, j)$ and $I_{w}(i, j)$ are the values of intensity at position $(i, j)$ of the original and watermarked image respectively and $I_{p e a k}$ is the peak value, equal to 255 for an image of $8 \mathrm{bpp}$.

If the image is divided in blocks it is possible to determine which block was altered in case this happened. The number of thresholds equals the number of blocks, and all of them must be part of the watermark, so they decrease the capacity. To determine by trial and error the values of the thresholds can be complicated and take long, specially in 16 bpp images. Genetic algorithms can be employed to optimize the solution.

\section{Genetic Algorithms in Watermarking}

The technique of searching for an optimal solution called GA (Genetic Algorithms) is inspired in the theory of biological evolution enunciated by Darwin [6]. GA have been used to solve problems of watermarking by different researchers. Between them, Shih and Woo [7] developed a robust watermarking system embedding in the DCT (Discrete Cosine Transform) particularly resistant to JPEG compression. They tried to minimize the round errors produced when converting real numbers into integers during the transformation. The initial chromosome is the difference between the initial image and the rounded image. Sikander $e t$ al. [8] proposed a method based on GA to increase the strength of the watermark embedded in the DCT domain. They tested the strength of the watermarked image to attacks as cropping, median and high-pass filters.

Nowadays, Arsalan, Malik and Khan [9] proposed the GA to determine the threshold in the compansion process to be applied in Xuan's algorithm [10].

In this work, the initial generation is composed by thresholds, one by block. The capacity is the pre-requisite . The fitness function used to evaluate the individuals has been the MSE. From one generation to the next, according to mechanisms of selection, crossover and mutation [11] [12] the thresholds change decreasing MSE. Because it is necessary to embed additional data to recover the original image, it could happen that the desired-effective payload can't be embedded because the effective-payload is higher. In this case the MSE 
Proc. of The Second Intl. Conf. On Advances In Computing, Control And Networking - ACCN 2015

Copyright $(\odot$ Institute of Research Engineers and Doctors, USA .All rights reserved.

ISBN: 978-1-63248-073-6 doi: 10.15224/ 978-1-63248-073-6-03

is considered Infinity. Once calculated the MSE, the PSNR is obtained.

MatLab (Version 2013b) has been used. The population size chosen was equal to 100 , and the number of generations 100. Roulette wheel ranking selection and tournament selection has been used alternatively without noticing significant differences between these methods, $2 \%$ was the elite rate, the crossover rate $80 \%$ and the mutation rate $1 \%$.

The process of selection, crossover and mutation continues until the termination condition (a low fixed MSE or the number of generations) is reached. Once selected the best or almost-best threshold matrix the process of embedding and extracting is tested.

\section{Experimental Results}

Some of the tested images are shown in Fig. 2 and in Fig. 3. Fig. 4 shows the comparison between the algorithm of Xuan that compresses the medium bitplane of the IWT and original Tian's algorithm. Tian's algorithm is clearly superior. Fig. 5 shows quality versus payload in 'lena.pgm' using Tian on quads and thresholds set by means of GA. We can observe that variations in the number of blocks do not alter substantially the results.

Tables I and II show the result of applying GA to optimize the imperceptibility using blocks to detect adulterations in the common images shown in Fig. 2. Table III shows the quality versus effective-payload for medical images. The ellipsis indicates that the effective payload can not be attained.

To increase the capacity of the method it is possible to repeat the algorithm varying the pivot when differences are calculated. This way the capacity can be duplicated and even triplicated.
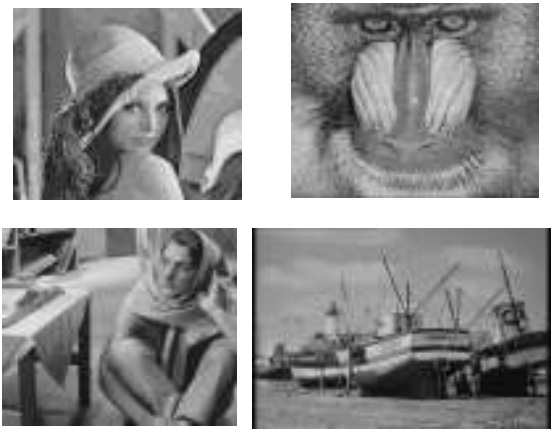

Figure 2. 'lena' 'baboon' 'barbara' 'boats'
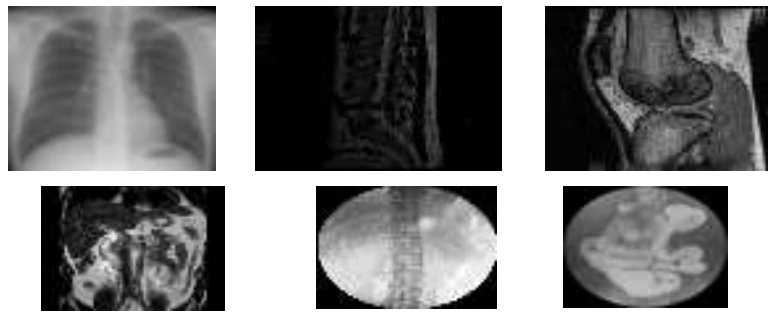

Figure 3. 'cr-chest', 'mr-spine', 'mr-knee', 'mr-abdo', 'cr-kidney', 'cr-intest'

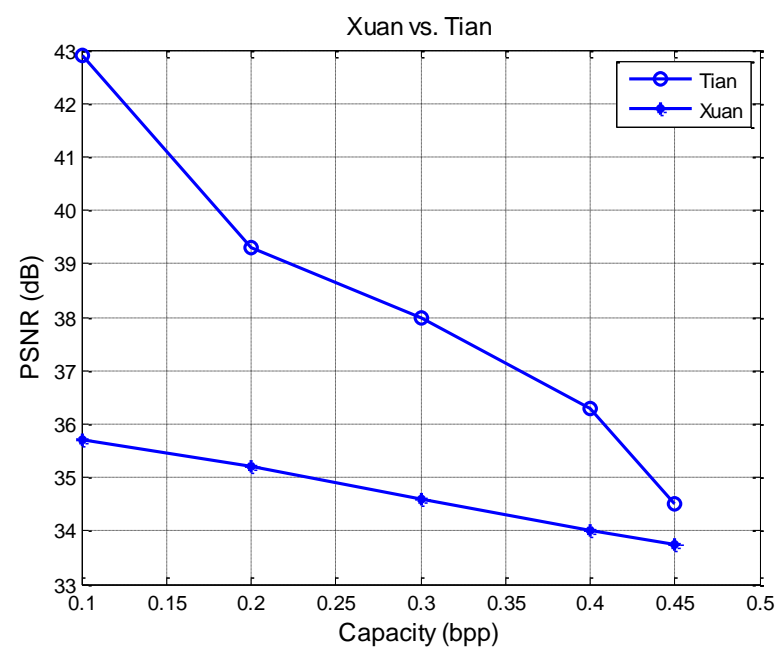

Figure 4. Original Tian vs. Xuan (Bitplane Compressed: 4)

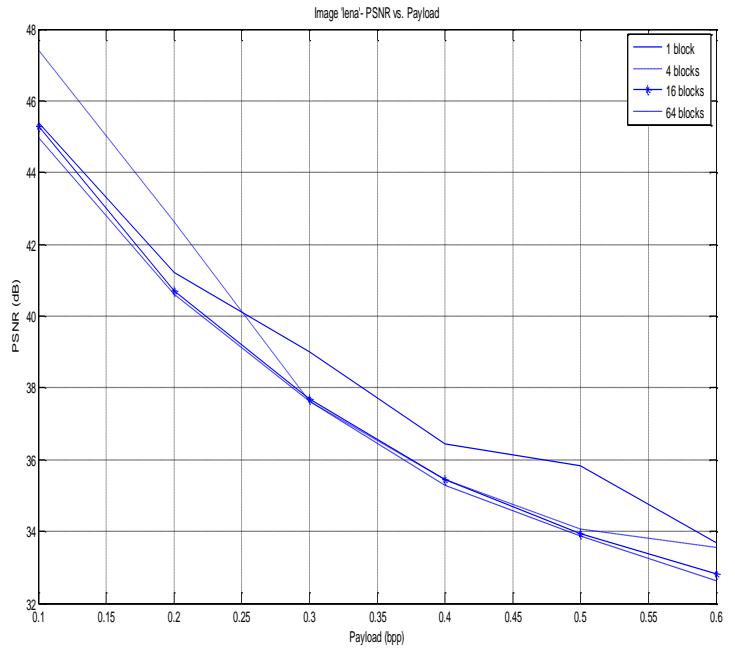

Figure 5. PSNR vs. Payload- 'lena'- Variable: Blocks

TABLE I. Quality vs. Capacity. Number of Blocks: 4

\begin{tabular}{|c|c|c|c|c|c|}
\hline $\begin{array}{c}\text { Payload } \\
\text { (bpp) }\end{array}$ & Quality & $\begin{array}{c}\text { lena } \\
(512 \times 512)\end{array}$ & $\begin{array}{c}\text { baboon } \\
(512 \times 512)\end{array}$ & $\begin{array}{c}\text { barbara } \\
(512 \times 512)\end{array}$ & $\begin{array}{c}\text { boats } \\
(576 \times 720)\end{array}$ \\
\hline \multirow{2}{*}{0,1} & PSNR(dB) & 47,42 & 31,84 & 38,61 & 47,43 \\
\hline & MSE & 1,18 & 42,63 & 8,95 & 1,17 \\
\hline \multirow{2}{*}{0,2} & PSNR(dB) & 42,64 & 28,03 & 36,17 & 41,30 \\
\hline & MSE & 3,54 & 102,28 & 15,69 & 4,82 \\
\hline \multirow{2}{*}{0,3} & $\operatorname{PSNR}(\mathrm{dB})$ & 37,64 & 26,63 & 31,64 & 37,80 \\
\hline & MSE & 11,19 & 141,16 & 45,28 & 12,98 \\
\hline \multirow{2}{*}{0,4} & PSNR(dB) & 35,44 & 25,10 & 30,23 & 36,60 \\
\hline & MSE & 18,59 & 201,37 & 61,68 & 14,27 \\
\hline \multirow{2}{*}{0,5} & PSNR(dB) & 34,08 & 24,47 & 27,57 & 34,41 \\
\hline & MSE & 25,44 & 201,37 & 113,62 & 23,55 \\
\hline \multirow{2}{*}{0,6} & PSNR(dB) & 33,55 & 24,09 & 26.97 & 32,58 \\
\hline & MSE & 28,87 & 258,28 & 130,49 & 35,85 \\
\hline \multirow{2}{*}{0,65} & PSNR(dB) & 32,85 & \multirow{2}{*}{$\begin{array}{c}\text { Not } \\
\text { Attained }\end{array}$} & 26,75 & 31,71 \\
\hline & MSE & 33,77 & & 137,68 & 43,83 \\
\hline \multirow{2}{*}{0,7} & PSNR(dB) & 32,43 & \multirow{2}{*}{$\begin{array}{c}\text { Not } \\
\text { Attained }\end{array}$} & \multirow{2}{*}{$\begin{array}{c}\text { Not } \\
\text { attained }\end{array}$} & 31,29 \\
\hline & MSE & 37,11 & & & 48,27 \\
\hline
\end{tabular}


Proc. of The Second Intl. Conf. On Advances In Computing, Control And Networking - ACCN 2015 Copyright $(\odot$ Institute of Research Engineers and Doctors, USA .All rights reserved.

ISBN: 978-1-63248-073-6 doi: 10.15224/ 978-1-63248-073-6-03

TABLE II. Quality vs. Capacity. Blocks 8x8

\begin{tabular}{|l|l|c|c|c|c|}
\hline $\begin{array}{c}\text { Payload } \\
\text { (bpp) }\end{array}$ & Quality & $\begin{array}{c}\text { lena } \\
(\mathbf{5 1 2 \times 5 1 2})\end{array}$ & $\begin{array}{c}\text { baboon } \\
\mathbf{( 5 1 2 \times 5 1 2 )}\end{array}$ & $\begin{array}{c}\text { barbara } \\
\mathbf{( 5 1 2 \times 5 1 2 )}\end{array}$ & $\begin{array}{c}\text { boats } \\
(\mathbf{5 7 6 x 7 2 0})\end{array}$ \\
\hline \multirow{2}{*}{0,1} & PSNR(dB) & 44,97 & 29,13 & 35,56 & 41,04 \\
\cline { 2 - 6 } & MSE & 2,07 & 78,60 & 18,06 & 5,11 \\
\hline \multirow{2}{*}{0,2} & PSNR(dB) & 40,62 & 26,40 & 34,78 & 38,54 \\
\cline { 2 - 6 } & MSE & 5,603 & 149,27 & 21,66 & 9,09 \\
\hline \multirow{2}{*}{0,3} & PSNR(dB) & 37,62 & 25,58 & 31,48 & 36,07 \\
\cline { 2 - 6 } & MSE & 11,24 & 179,94 & 26,29 & 16,09 \\
\hline \multirow{2}{*}{0,5} & PSNR(dB) & 33,88 & 24,67 & 27,94 & 32,99 \\
\cline { 2 - 6 } & MSE & 26,60 & 224,77 & 104,32 & 32,63 \\
\hline \multirow{2}{*}{0,6} & PSNR(dB) & 32,61 & 24,09 & 27,03 & 31,89 \\
\cline { 2 - 6 } & MSE & 35,62 & 254,59 & 128,72 & 42,05 \\
\hline
\end{tabular}

Table III. Quality vs. Capacity. Blocks 16x16

\begin{tabular}{|c|c|c|c|c|c|c|}
\hline \multirow[b]{2}{*}{$\begin{array}{l}\text { Pay } \\
\text { load } \\
\text { bpp }\end{array}$} & \multicolumn{6}{|c|}{$\begin{array}{c}\text { PSNR } \\
\text { (dB) }\end{array}$} \\
\hline & $\begin{array}{c}\text { cr- } \\
\text { chest } \\
440 \times 440 \\
16 \text { bpp }\end{array}$ & $\begin{array}{c}\mathrm{mr} \\
\text { spine } \\
512 \times 512 \\
16 \mathrm{bpp} \\
\end{array}$ & $\begin{array}{c}\mathrm{mr} \\
\text { knee } \\
256 \times 256 \\
16 \mathrm{bpp} \\
\end{array}$ & $\begin{array}{c}\mathrm{mr} \\
\text { abdo } \\
256 \times 256 \\
16 \mathrm{bpp} \\
\end{array}$ & $\begin{array}{c}\text { cr } \\
\text { kidney } \\
512 \times 512 \\
8 \text { bpp } \\
\end{array}$ & $\begin{array}{c}\text { cr } \\
\text { intest } \\
512 \times 512 \\
8 \text { bpp } \\
\end{array}$ \\
\hline 0,1 & 88,48 & 75,78 & 86,81 & 70,10 & 45,62 & 44,61 \\
\hline 0,2 & 86,04 & 75,25 & 82,94 & 68.09 & 44,24 & 42,31 \\
\hline 0,3 & 84,74 & 75,16 & 80,67 & 66,75 & 43,72 & $39,, 93$ \\
\hline 0,35 & 84,21 & 74,42 & 79,81 & 66,39 & 43,29 & 39,23 \\
\hline 0,4 & 83,67 & 72,80 & 79,11 & --- & --- & --- \\
\hline 0,45 & 83,23 & 72,53 & 78,58 & --- & --- & --- \\
\hline 0,5 & 82,83 & --- & 78,88 & --- & --- & --- \\
\hline 0,6 & 81,99 & --- & 77,18 & --- & --- & --- \\
\hline 0,7 & 81,39 & --- & 76,77 & --- & --- & --- \\
\hline
\end{tabular}

Fig. 6 shows the comparison between the proposed implementation of GA against Xuan with GA, both of them applied on 'lena'. Xuan shows better quality than Tian quads. The disadvantage of Xuan's algorithm is that it sometimes needs the previous compression of the histogram to avoid overflow or underflow, while the proposed algorithm does not, doing a simple operation mathematical prevents over and underflow. In 'lena.pgm' it is not necessary to compress the histogram, but 'baboon.pgm' only reaches a payload of 0,1 bpp without compression of the histogram. Applying more than once the algorithm is always possible.

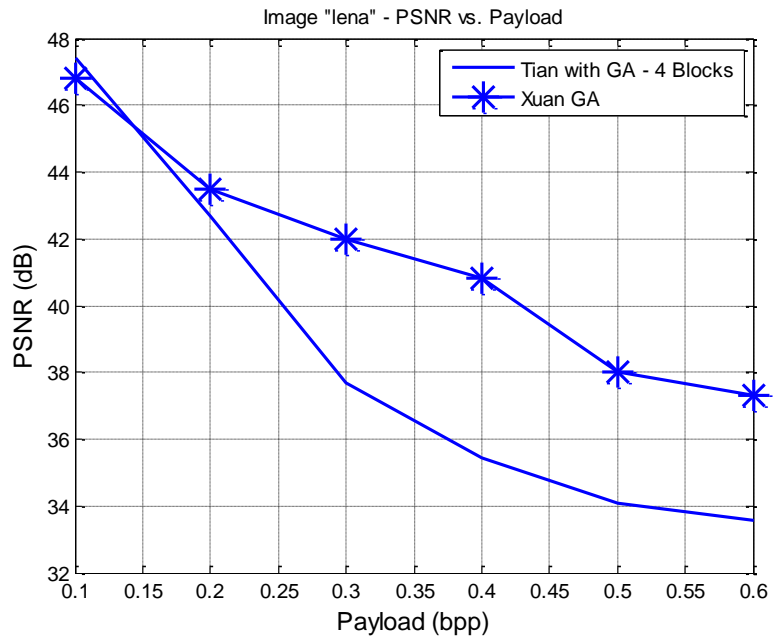

Figure 6. Tian vs. Xuan with GA. 'lena.pgm’
TABLE IV- Algorithm of Xuan

\begin{tabular}{|c|c|c|c|c|}
\hline \multirow{3}{*}{$\begin{array}{c}\text { Payload } \\
\text { (bpp) }\end{array}$} & \multicolumn{4}{|c|}{ Image } \\
\hline & \multicolumn{2}{|c|}{$\begin{array}{c}\text { lena } \\
512 \times 512 \times 8 \\
\end{array}$} & \multicolumn{2}{|c|}{$\begin{array}{c}\text { baboon } \\
512 \times 512 \times 8 \\
\end{array}$} \\
\hline & $\operatorname{PSNR}(\mathrm{dB})$ & MSE & $\operatorname{PSNR}(\mathrm{dB})$ & MSE \\
\hline 0,04 & 50,48 & 0,58 & 31,71 & 43,82 \\
\hline 0,1 & 46,80 & 1,54 & \multirow{6}{*}{\multicolumn{2}{|c|}{$\begin{array}{l}\text { Overflow- } \\
\text { Histogram } \\
\text { Compression } \\
\text { is Needed }\end{array}$}} \\
\hline 0,2 & 43,61 & 2,82 & & \\
\hline 0,3 & 42,06 & 4,04 & & \\
\hline 0,4 & 40,81 & 5,28 & & \\
\hline 0,5 & 38,04 & 10,20 & & \\
\hline 0,6 & 37,35 & 11,94 & & \\
\hline
\end{tabular}

The same disadvantage is noticed in medical images as it can be appreciated in Table $\mathrm{V}$. The compression of the histogram takes time and decreases the quality, and it can't be determined in advance.

TABLE V. Algorithm of Xuan- Medical Images

\begin{tabular}{|c|c|c|c|c|c|c|}
\hline \multirow{2}{*}{$\begin{array}{l}\text { Pa } \\
\text { yload } \\
\text { (bits) }\end{array}$} & \multicolumn{2}{|c|}{$\begin{array}{c}\text { cr-chest } \\
440 \times 440 \\
16 \text { bpp } \\
\end{array}$} & \multicolumn{2}{|c|}{$\begin{array}{c}\text { mr-knee } \\
256 \times 256 \\
16 \text { bpp } \\
\end{array}$} & \multicolumn{2}{|c|}{$\begin{array}{c}\text { mr-spine } \\
512 \times 512 \\
16 \text { bpp } \\
\end{array}$} \\
\hline & $\begin{array}{c}\text { Capacity } \\
\text { (bpp) }\end{array}$ & $\begin{array}{c}\text { PSNR } \\
\text { (dB) }\end{array}$ & $\begin{array}{c}\text { Capacity } \\
\text { (bpp) }\end{array}$ & $\begin{array}{l}\text { PSNR } \\
(\mathrm{dB})\end{array}$ & $\begin{array}{c}\text { Capacity } \\
\text { (bpp) }\end{array}$ & $\begin{array}{c}\text { PSNR } \\
(\mathrm{dB})\end{array}$ \\
\hline 1024 & 0,005 & 83,71 & 0,015 & 91,73 & 0,004 & 121 \\
\hline 2048 & 0,010 & 82,77 & 0,03 & 90,68 & 0,008 & 118 \\
\hline 4096 & 0,015 & 83,30 & 0,06 & 87,07 & \multicolumn{2}{|c|}{$\begin{array}{c}\text { Histogram } \\
\text { Compression } \\
\text { Needed }\end{array}$} \\
\hline
\end{tabular}

The perfomance of the algorithms of watermarking can be also expressed in terms of SSIM (Structural Similarity Index) that considers the Human Visual System (HVS) [13]. An image as baboon with a low PSNR has a good value of SSIM, because the HVS does not detect the mark because of the high local variance that this image presents. So this index can also be used as the fitness function.

\section{v. Conclusions}

An intelligent watermarking approach has been developed. It can be specially applied in medical images because they can be typified and once the thresholds are determined they can be used for all the medical images of the same type.

The use of GA has proved to be particularly useful to attain the effective capacity. After a few number of generations in images as baboon or medical images with high local variance where it is difficult to embed high payloads because the differences are high the desired effective payload is achieved.

As the original image is recovered it is not necessary to avoid the Region of Interest (ROI) in medical images nevertheless this can also be done thereby increasing the quality of the watermarked image for common users.

The running of the algorithm takes time but once the typical thresholds of medical images are found, it is not 
necessary to repeat the procedure. Parallel processing must be applied to reduce the running time.

\section{References}

[1] Z. Ni., Q. Shi., N. Ansari. y W. Su. "Reversible Data Hiding”, IEEE Transactions on Circuits and Systems for Video Technology, Vol. 16, $\mathrm{N}^{\circ} 3,2006$, pp. 354-361.

[2] G. Xuan, J. Zhu, J. Chen, Y. Shi, Z. Ni y W. Su, "Distortionless data hiding based on integer wavelet transform", Electronics Letters, 2002, pp. 1646-1648.

[3] J. Tian, "Reversible Data Embedding Using a Difference Expansion", IEEE Transactions on Circuits and Systems for Video, Vol. 13, $\mathrm{N}^{\circ} 8$, 2003, pp. 890-896.

[4] S. Weng, Y. Zhao, J. Pan y R. Ni, "A novel reversible watermarking based on an integer transform", International Conference on Image Processing ICIP, Vol. 3, 2007, pp. 241-243.

[5] A. M. Alattar, "Reversible Watermark Using Difference Expansion of Quads", Proceedings of IEEE International Conference on Acoustics, Speech and Signal Processing, Vol. 3, 2004, pp. 377-380.

[6] J. Holland, "Genetic Algorithms“, Scientific American, pp.67-72, 1992.

[7] F. Y. Shih, Y. Ta Wu, "Robust Watermarking and Compression for Medical Images Based on Genetic Algorithm", Journal of Information Sciences, 2005, pp. 200-216.

[8] B. Sikander, M. Ishtiqa, M. Arfan, M. Tariq, A. Mirza, "Adaptive Digital Watermarking of Images Using Genetic Algorithm", 2010 ICISA, International Conference on Information Science and Applications, 2010, pp. 1-8.

[9] M. Arsalan, S. Malik, A. Khan, "Intelligent Reversible Watermarking in Integer Wavelet Domain for Medical Images", The Journal of Systems and Software, Ed. Elsevier, Vol. 85, 2012, Issue 4, pp.883-894.

[10] G. Xuan, Y. Q. Shi, C. Yang, Y. Zheng, D. Zou. P. Cha, "Lossless Data Hiding Using Integer Wavelet Transform and Threshold Embedding Technique", Multimedia and Expo. ICME 2005. IEEE International Conference, pp. 1520 - 1523, 2005.

[11] R. L. Haupt, S. E. Haupt, "Practical Genetic Algorithms", Ed. WileyInterscience, $2^{\text {nd }}$ E. Edition, 2004.

[12] M. Affenzeller, S. Winkler, S. Wagner, A. Beham, "Genetic Algorithms and Genetic Programming, Modern Concepts and Practical Applications", CRC Press, Taylor and Francis Group, LLC, 2009.

[13] Z. Wang, A. Bovik, H.Sheikh, E. Simoncelli. "Image Quality Assurement: from Error Visibility to Structural Similarity". IEEE Transactions on Image Processing, Vol. 13, N4, 2004, pp. 600-612.

About Author (s):

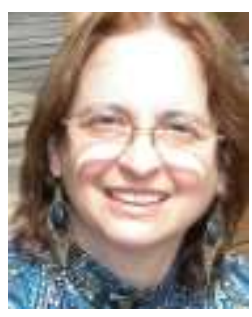

Doctor in Engineering Sciences, ElectricianElectronic Engineer, University National of Cordoba, Argentina.

Associate Professor Full Dedication in Data Communication, University National of Cordoba., Faculty of Exact, Physical and Natural Sciences, Computing Department.

Interested in Image Processing, Networking, Data Communication, University Teaching. 